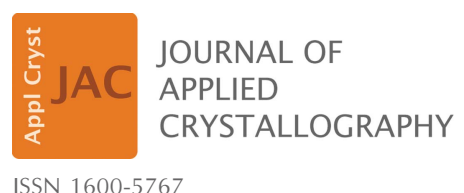

ISSN 1600-5767

Received 4 October 2015

Accepted 8 October 2015

Keywords: deviatoric elastic strain; lattice orientation; digital image correlation; Laue microdiffraction.
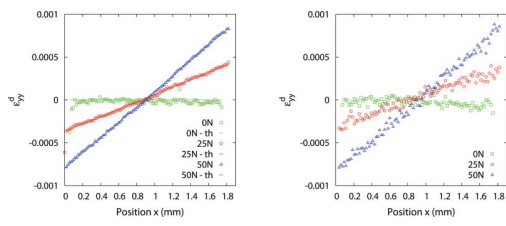

(C) 2015 International Union of Crystallography

\section{Accurate strain determination from digital image correlation of Laue diffraction spots}

\author{
András Borbély* \\ Ecole Nationale Supérieure des Mines, SMS-EMSE, CNRS:UMR 5307, LGF, 42023, Saint-Etienne, Cedex 2 France. \\ *Correspondence e-mail: andras.borbely@mines-stetienne.fr
}

Accurate strain/stress characterization has important implications in practice, where it is typically connected with safe operation of engineering components. Improving the accuracy and spatial resolution of the measurement techniques can be therefore very beneficial for materials science by helping the understanding of complex material behaviour and the development of novel materials with improved properties. Strain measurement by diffraction methods is based on Bragg's law, which in the case of a strained crystal predicts a shift of the diffraction peaks compared to their position measured on a reference state. Classically, this shift is determined as the difference between the corresponding absolute peak positions, which requires in many cases accurate knowledge of

(i) the geometrical setup and its stability,

(ii) the calibration sample, and

(iii) a well matching mathematical model of the intensity distribution.

In view of this large number of parameters it is intriguing to ask what the accuracy of today's X-ray diffraction (XRD) methods is and which are the possible ways of improvement. Limiting our survey to methods able to determine the strain tensor at micrometre scale, one can rapidly find that the error of most strain tensor components is about a few times $10^{-4}$. Transformed to stress (using Young's modulus of $\sim 100 \mathrm{GPa}$ ) this represents an uncertainty of tens of $\mathrm{MPa}$, which might be too large for the study of certain phenomena, such as for example the plastic yield of metals. Therefore, efforts are continuously undertaken to ameliorate the precision of the evaluation methods. The majority of the solutions, however, concern point (i), suggesting the use of large area detectors with many pixels and without distortion, or fixing instabilities of the wavelength or micro-spot position by applying more stable optical elements. Progress in this case depends on technical advancement, which sometimes might be slow compared to the needs of the scientific community. Increasing strain/stress accuracy when working with conventional setups needs, therefore, a paradigm change, i.e. a method different from the classical approach based on absolute peak positions.

Two recently published articles by Petit et al. (2015) and Zhang et al. (2015) break the tradition and introduce evaluation schemes based on the shift of diffraction peaks determined by digital image correlation (DIC). This new idea, which plays a central role in experimental mechanics, is applied in both papers to white-beam Laue microdiffraction, a popular method often analysed in terms of error sources impeding accurate determination of the deviatoric strain tensor (Hofmann et al., 2011; Poshadel et al., 2012). As argued by Petit et al. (2015), the gain in accuracy of the Laue-DIC method is related to two factors: $(a)$ the smaller uncertainty of the peak shift determined by DIC and $(b)$ the higher sensitivity of the merit function expressing peak positions as a function of reference cell parameters, compared to the sensitivity of the gradient of the merit function, the latter appearing in the formulation of Laue-DIC. From the point of view of the error sources highlighted above it becomes clear that the new approach eliminates the uncertainties related to the choice of an adequate mathematical model describing the experimental intensity distribution. The authors emphasize that relatively good fits can be obtained for the spots of the non-deformed reference specimen using Gaussian or Pearson VII profiles, but already as the specimen is strained, peak quality deteriorates and the uncertainty in peak position increases above 0.1 pixel, characteristic for the reference peaks. Compared to this the uncertainty of DIC is one order of magnitude 
smaller. The paper explains in a clear and intuitive way why the innovative idea of applying DIC to diffraction peaks works so well for Laue spots. After analysing its robustness to image noise and the stability of the setup, the authors apply the method to the determination of the deviatoric strain tensor in a silicon single crystal strained in four-point bending. Fig. 1(a) shows the variation of the deviatoric strain component along the direction of the bending force (at the middle of the specimen and normal to the neutral zone; for details see Petit et al., 2015) at three applied loads. Excellent agreement between experimental results and theoretical prediction can be stated, the deviation between them being of the order of $10^{-5}$. Compared to the results obtained from the analysis of the same diffraction images according to the classical procedure (Fig. 1b) the major improvement of Laue-DIC, showing significantly reduced scatter, is quite evident.

Usually a strain accuracy of $10^{-5}$ suffices for most experimental tests aiming to check the prediction of a given physical model or just to characterize material response to an applied load. However, recalling the three main sources of error of the classical method we should acknowledge that challenges related to the stability of the geometrical setup and calibration sample are not solved. On the basis of their experience with Laue-DIC, Zhang et al. (2015) analysed this problem more thoroughly and developed the 'enhanced Laue-DIC' method, which at the expense of a more complex numerical procedure directly accounts for eventual instabilities of the setup and for the reference state. This simplifies tremendously the practical implementation of the technique. Another advantage is that 'enhanced' Laue-DIC calculates the deviatoric elastic strain, while original Laue-DIC calculates only the elastic strain increment. The term 'enhanced' refers not to more accurate strains but to the more general character conveyed by the increased number of parameters resulting from the analysis. While the original Laue-DIC method comprises only eight unknown parameters (describing the normalized lattice matrix of the current state), the enhanced version contains 24 unknowns, from which $8+8$ are related to the lattice matrices of the current and reference states, and $4+4$ describe the geometrical setup corresponding to measurement conditions in the two states. Fitting 24 unknowns is not obvious, but applying different optimization schemes the authors succeed in obtaining an accuracy for the deviatoric strain components that is similar to or just slightly worse than what they have obtained with original Laue-DIC.

Finally, it is worth discussing why DIC is applicable to determining diffraction spot displacement. It is known that the method exploits the resemblance between a reference and a current pattern, which in the case of diffraction spots is determined by the corresponding distribution of the intensity values (peak shape). Trying to understand the analogy with the classical method, the simplest question we can ask is why the distance between two peak centres (both associated with an average unit cell) is identical to the displacement quantified by DIC, which geometrically represents the displacement of a rectangular 'zone of interest' centred on the spots of interest. In the easiest case, when peak shape does not change much (being only shifted), the answer is obvious. In physical terms Petit et al. (2015) argue that spot resemblance is guaranteed by the small difference between the substructures of the irradiated volumes in the reference and current states. This was evidently the case for the elastically bent $\mathrm{Si}$ single crystal, when DIC delivered excellent results with a simple shape function allowing only rigid body motion. But, what is happening and how accurate will the results be when the spot shape changes? An intriguing example is that of plastically deformed crystals when it is known that Laue spots reveal a well pronounced streaking (Barabash et al., 2001). The authors argue that DIC can handle this challenge too, and for crystals containing orientation gradients they suggest the use of specific 'connected' shape functions, which could detect similarity in spot shape. Evidently, accurate results for such

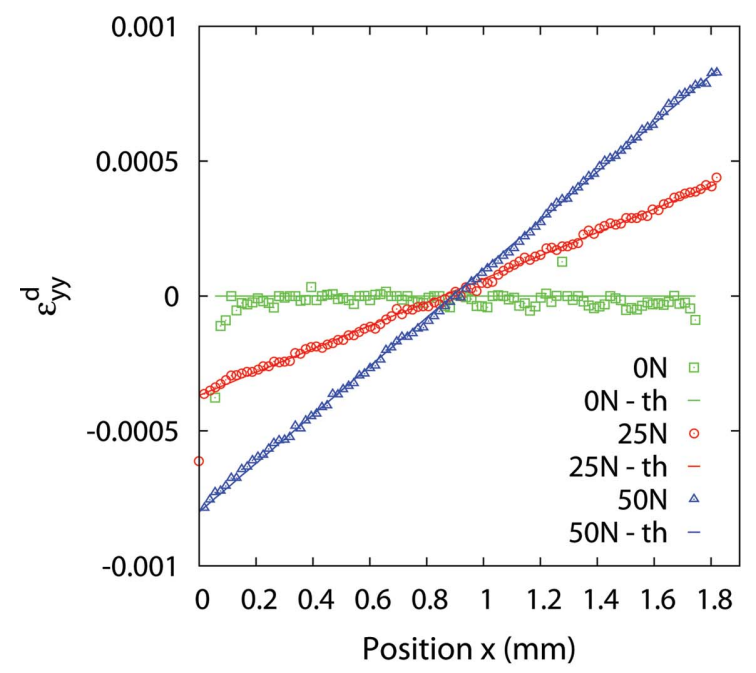

(a)

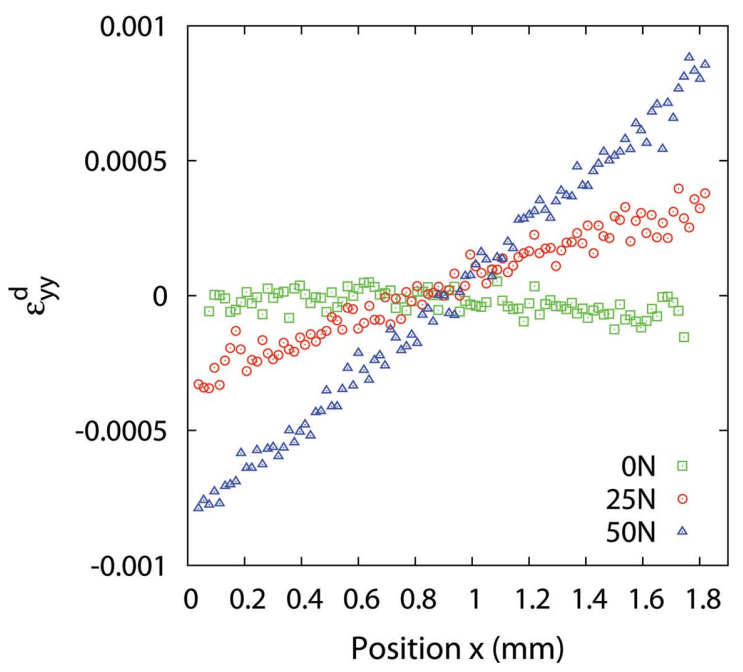

(b)

Figure 1

(a) Comparison between the deviatoric strain component obtained with the Laue-DIC method and the prediction of the asymptotic beam theory (denoted by 'th') at three external loads. (b) The same data set evaluated according to the classical approach based on peak fitting (reproduced from Petit et al., 2015). 


\section{scientific commentaries}

complicated cases will be very helpful for the materials science community, which is looking forward to a related publication.

\section{References}

Barabash, R., Ice, G. E., Larson, B. C., Pharr, G. M., Chung, K.-S. \& Yang, W. (2001). Appl. Phys. Lett. 79, 749-751.
Hofmann, F., Eve, S., Belnoue, J., Micha, J.-S. \& Korsunsky, A. M. (2011). Nucl. Instrum. Methods Phys. Res. Sect. A, 660, 130-137. Petit, J., Castelnau, O., Bornert, M., Zhang, F. G., Hofmann, F., Korsunsky, A. M., Faurie, D., Le Bourlot, C., Micha, J. S., Robach, O. \& Ulrich, O. (2015). J. Synchrotron Rad. 22, 980-994.

Poshadel, A., Dawson, P. \& Johnson, G. (2012). J. Synchrotron Rad. 19, 237-244.

Zhang, F. G., Castelnau, O., Bornert, M., Petit, J., Marijona, J. B. \& Planchera, E. (2015). J. Appl. Cryst. 48, 1805-1817. 